\title{
MULTISENSOR FAULT DETECTION AND ISOLATION USING KULLBACK LEIBLER DIVERGENCE : APPLICATION TO DATA VIBRATION SIGNALS
}

\author{
Claude DELPHA*, Demba DIALLO ${ }^{\dagger}$, Tianzhen $W A N G^{\ddagger}$, Jie LIU ${ }^{\S}$, Zelig $L I^{\S}$ \\ ${ }^{*}$ Laboratoire des Signaux et Systèmes (L2S), \\ CNRS - CentraleSupelec - Univ. Paris-Sud, Gif sur Yvette, France \\ $\dagger$ Group of Electrical Engineering of Paris (GeePs), \\ CNRS - CentraleSupélec - Univ. Paris-Sud - UPMC , Gif sur Yvette, France \\ ${ }^{\ddagger}$ Shanghai Maritime University, Department of Electrical Automation, Shanghai, China \\ $\S$ Carleton University, Mechanical and Aerospace Engineering, Ottawa, Canada
}

\begin{abstract}
In this paper we develop a fault detection and isolation method based on data-driven approach. Data-driven methods are effective for feature extraction and feature analysis using statistical techniques. In the proposal, the Principal Component Analysis (PCA) method is used to extract the features and to reduce the data dimension. Then, the KullbackLeibler Divergence (KLD) is used to detect the fault occurrence by comparing the Probability Density Function of the latent scores. The faulty sensor is isolated thanks to a linear combination of the original measurements with binary coefficients denoted as Z-decomposition. The proposed approach is experimentally verified with vibration signals used for monitoring bearings in electrical machines.
\end{abstract}

\section{Nomenclature}

CWRU Case Western Reserve University

FDD Fault Detection and Diagnosis

KLD Kullback-Leibler Divergence

KLI Kullback-Leibler Information

MC Monte Carlo

PCA Principal Component Analysis

PDF Probability Density Function

rpm round per minute

\section{INTRODUCTION}

Fault detection and diagnosis has received increasing attention since the last two decades. The detection of faults at an early stage, their isolation and the analysis of their causes are essential to ensure the safety, reliability and good performances of the application.

For example, electrical rotating machines usually operate by means of bearings which are among the most critical components [1]. The quality of the motor system operation is closely related to the performance of bearing assembly. Among the state-of-the-art, vibration monitoring is asserted to be one of the most effective and practical techniques to detect and diagnose bearing faults [2]. Although bearing vibration signals, which cover displacement, velocity and acceleration signals, are rarely straightforward and may contain vibration components generated by various mechanical and electromagnetic forces, they provide the most salient information for the early detection of bearing faults. Unfortunately, the vibration sensors may be exposed to failures in hard industrial conditions. Therefore, it is necessary to detect these failures and to estimate their amplitudes in order to correct the measurements.

Just as any dynamic system, a sensor fails if a failure occurs in any of its components including the sensing device, transducer, signal processor, or data acquisition equipment. An abrupt failure in the sensor can be caused by a power failure or corroded contacts, while an incipient failure such as drift and precision degradation can be caused by deterioration in the sensing element. As defined in [3], both an abrupt and an incipient failure can cause non-permitted deviation from the characteristic property in a sensor, which leads to inaccurate measurements from the monitored system. Consequently, a faulty sensor can cause process performance degradation, process shut down, or even worse in a safety critical system. In fact, the problem of instrument fault detection, identification and accommodation has already received extensive attention in both industrial and academic fields. Nevertheless, the detection of incipient sensor failures that is important for critical information to diagnose and control systems has received limited attention in literature [4].

A conventional engineering method for sensor validation is to check and recalibrate a sensor periodically according to a set of predetermined procedures. Although this method has been widely implemented in industry for detecting abrupt 
sensor failures, it is not able to accomplish continuous assessment of a sensor, and thus is not effective in detecting its incipient failure. Moreover, due to their ever increasing number, it has become cost ineffective and even infeasible to check all sensors periodically. Therefore, significant efforts have been made for the development of more systematic methods, which can be generally categorized into hardware and analytical redundancy approaches [5].

Different from model-based approaches that require accurate analytical multiphysics-based description of the target system, data-driven methods, also known as process history based methods, require the availability of sufficient data [6]. Various methods have been developed to establish the knowledge database for the underlying system by extracting characteristic features directly from its past performance data. We can find in this approach different methods like multivariate statistical methods [6], Bayesian belief networks, and neural networks [7].

In this paper, we adopt a data-driven approach [8] using descriptive features within the Principal Component Analysis (PCA) [9], framework combined with multivariate statistical techniques to develop an efficient fault detection and isolation method.

PCA-based monitoring methods can easily handle high dimensional, noisy and highly correlated data generated from industrial processes, and provide superior performance compared to univariate methods [9]. In addition, these process monitoring techniques are attractive for industrial practical processes because they only require a good historical data set of healthy operation, which are easily obtainable for computer-controlled industrial processes. PCA-based monitoring methods and their extensions have been successfully applied in a wide range of applications and industries, such as in chemical processes, air quality, water treatment, aerospace, agriculture, automotive, electronics, energy, manufacturing, medical devices, and many others [10].

It has already been shown, that in the PCA framework, the Kullback-Leibler Divergence (KLD) [11] is conceptually more straightforward and also more sensitive for the fault detection of incipient faults [12] than the usual detection indices, like the Hotelling $\mathbf{T}^{2}$ and squared prediction error (SPE).

In reliability studies, because failure times are defined only for times $>0$, the most common families of distributions used in place of the Normal distribution (truncated at the initial time value times $=0$ ) are the Exponential, the Weibull and the Gamma distributions. It has been established that Gamma distributed data are particularly suitable. An example of application is given by Nguyen et al. in [13].

The paper is organised as follows. In section 2, the fault detection and isolation procedures are described. In section
3 using numerical data the results of fault detection and fault isolation are presented. Finally the fault diagnosis procedure is applied to electrical machines vibration signals experimentally acquired from 8 accelerometers. A conclusion in section 4 closes the paper.

\section{FAULT DETECTION AND ISOLATION PROCEDURE}

The fault detection and diagnosis (FDD) procedure for complex systems health monitoring can be divided in three main operations as displayed in Figure 1: first, Fault Detection, second, Fault Isolation, and the last Fault Estimation. For FDD process, each operation can be done through 4 main steps (Fig.2): the Data modelling, the Preprocessing, the Feature extraction and the Feature analysis. In this work a data driven approach is considered. Therefore, the first step is the information extraction to obtain a data-based description of the process. In the following we describe the content of the detection and isolation operations with their different steps.

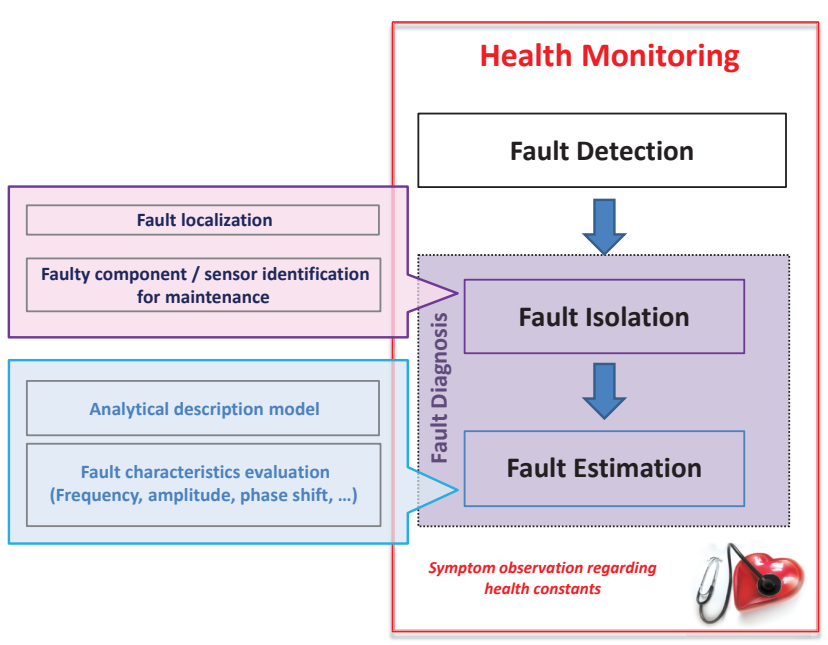

Fig. 1. Fault Detection and Diagnosis general scheme

\subsection{Fault Detection}

Fault detection plays a key role in enhancing today's technological systems high demands for performance and security. In such systems, minor faults can result in catastrophic consequences. For that reason, their is a huge need for very sensitive fault detection and diagnosis (FDD) methods. Such methods must be insensitive to the environment evolution (noise, temperature,...) but also to input changes. Nevertheless, they have to be very sensitive to the fault severity and allow early detection.

In this paper, as said before, we adopt a statistical method 
for incipient fault detection. The general procedure for data driven statistical monitoring is to collect a large number of healthy data samples used as the reference data set. All new measured data are then compared to the healthy ones to check whether an abnormal behavior occurs.

So, once the PCA's model is established, a reference probability distribution is estimated for each latent score. Then for each new set of observations, the associated latent scores are calculated through the PCA's model and their probability density functions (PDF) are estimated. Then, the KLD is used to measure the dissimilarities between the probability density functions of healthy data and measured ones.

\subsubsection{Fault Modeling}

Let's consider a database $X$ containing the information describing a complex system with $m$ independent variables and $N$ observations (measurements) for each variable. The original data are arranged such as $X=\left(x_{1}, \ldots, x_{j}, \ldots, x_{m}\right)$ where the vector for the $j^{\text {th }}$ independent variable can be written as $x_{j}=\left[x_{1 j}, \ldots, x_{i j}, \ldots, x_{N j}\right]^{\prime}$. Note that the superscript ' denotes the transpose operation symbol for the matrix.

Hereafter, we consider a gain fault $G=1+a$, such as the faulty signal is $x_{j}=G \times x_{i}$. Faulty components are therefore proportional to the reference signals. We assume that the considered fault occurs only on one descriptive feature (variable) among the $m$ measured ones. The fault affecting the $j$ th variable $x_{j}$ can be written as:

$$
f_{a}=a \times\left[\left[\begin{array}{l}
x_{1 j}^{*} \\
\vdots \\
\vdots \\
x_{N j}^{*}
\end{array}\right]+\left[\begin{array}{l}
v_{1 j} \\
\vdots \\
\vdots \\
v_{N j}
\end{array}\right]\right]
$$

Then $x_{j}=\left(x_{j}^{*}+v_{j}\right)+f_{a}$ where $f_{a}$ is the fault component, $a$ is the fault amplitude parameter, $x_{j}^{*}$ is the $j$ th reference signal which will be affected by the fault, and $v_{j}$ is the measurement noise. With such signal fault and noise modelling, we propose to study the Kullback-Leibler Divergence (KLD) for the fault detection.

Note that the superscript $*$ is placed for identifying the healthy and noise-free signals.

\subsubsection{Principal Component Analysis}

The goal of PCA is to compute the data in a new reduceddimension workspace highlighting the similarities between the data [9]. For this purpose, the original data matrix $X$ can be centered to compute the Principal Component Analysis (PCA) and obtain the scores matrix $T$ such as $T=\bar{X} P$, where $P$ is the loading eigenvectors matrix such as $P=\left(p_{1}, \ldots, p_{j}, \ldots, p_{l}, \ldots, p_{m}\right)$ and $\bar{X}$ is the centered data matrix.
The matrix $T=\left(t_{1}, \ldots, t_{j}, \ldots, t_{l}, \ldots, t_{m}\right)$ is also composed of $m$ variables and the $j^{t h}$ one is written as $t_{j}=\left[t_{1 j}, \ldots, t_{i j}, \ldots t_{N j}\right]^{\prime}$. Due to PCA, the score variables $t_{j}$ are linear combination of the original ones $x_{j}$ weighted by the eigenvectors $p_{j}$ where $p_{j}=\left[p_{1 j}, \ldots, p_{q j}, \ldots p_{m j}\right]^{\prime}$. The $p_{m j}$ elements are then the eigenvalues denoted $\lambda$ in the following.

\subsubsection{Kullback Leibler Divergence}

The divergence between the probability density functions (PDFs) of healthy and test data can be achieved by the KLD computation between the two distributions [11].

For discrimination between two continuous probability density functions (PDFs) $f(r)$ and $g(r)$ of a random variable $r$, the Kullback-Leibler Information (KLI) is defined as:

$$
I(f \| g)=\int f(r) \log \frac{f(r)}{g(r)} d r .
$$

The KL Divergence (KLD) is then defined as the symmetric version of the KL Information [11]:

$$
K L D(f, g)=I(f \| g)+I(g \| f)
$$

For arbitrary distributions $f$ and $g$, (2) can be numerically approximated using Monte Carlo (MC) simulation. The Monte Carlo method expresses (2) as the expectation of $\log (f / g)$, under the PDF $f$. Using $n_{s}$ i.i.d samples $\left\{z_{i}\right\}_{1}^{n_{s}}$ drawn from $f$, it consists in calculating:

$$
K \widehat{L} D(f, g)=D_{M C}(f, g)=\frac{1}{n_{s}} \sum_{i=1}^{n_{s}} \log \frac{f\left(z_{i}\right)}{g\left(z_{i}\right)}
$$

With such approximation, the estimation error distribution is normal with variance $\sigma_{M C}^{2}$ and zero mean $\left(\sim \mathcal{N}\left(0, \sigma_{M C}^{2}\right)\right)$ such as $\sigma_{M C}^{2}=\frac{1}{n_{s}} \mathbb{E}\left([\log (f / g)]^{2}\right)$. More the set of samples $n_{s}$ will be larger, smaller will be the Monte Carlo estimation error.

Therefore, the KLD's value obtained is compared to a threshold to make the decision: healthy or faulty. The setting of the threshold depends on the noise level [14].

The main steps of this fault detection procedure are shown in the flowchart depicted in Fig.2.

\subsection{Fault isolation}

Once a fault is detected, the faulty sensor must be identified. It is assumed that there is a single fault case.

The first idea was to use the PCA for isolation as it has already been used for fault detection. As a rule, knowing that each variable has a different contribution in every principal component (every principal component is a linear combination of the $m$ variables), a fault affecting one variable, will not have the same contribution on all the principal components. 


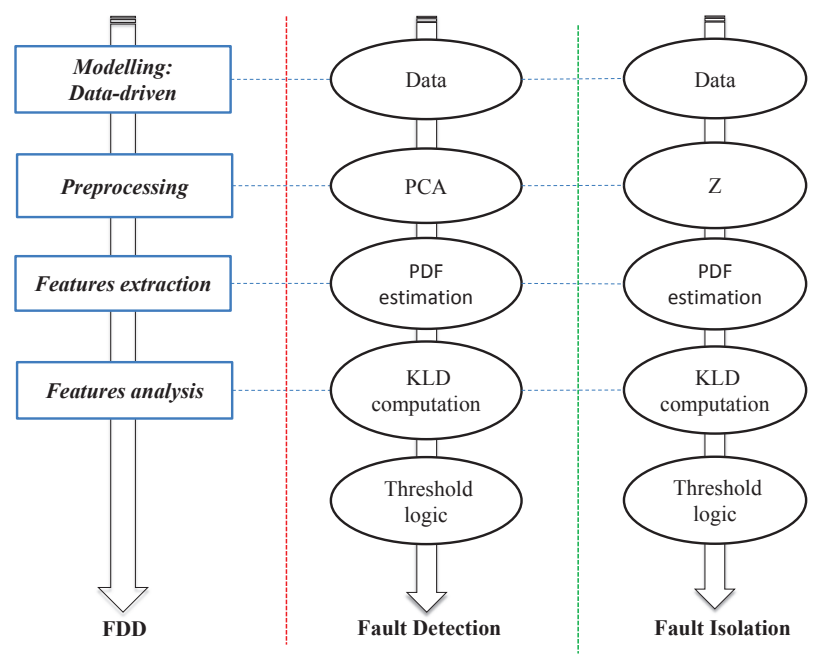

Fig. 2. Fault Detection and Diagnosis flowchart

For example, a small fault affecting the variable $x_{j}$, can only be detected in the principal components in which this variable is significantly represented.

In this original space, the fault isolation could be ambiguous because the contribution of the fault to the different principal components depends on the fault severity. Therefore the signature is no longer unique. To fix this issue, we propose to transform the initial $m$ variables into $K_{z}$ new ones $\left(K_{z}<m\right)$ obtained as linear combinations of the initial variables with binary coefficients. This operation is called the Z-decomposition.

The $K_{z}$ value is obtained as follows :

$$
K_{z}=\frac{\log (m)}{\log (2)}+1
$$

For example for $m=7$, we obtain $K_{z}=3$. We show how to create the combination matrix in the table below:

Table 1. Fault signatures

\begin{tabular}{cccccccc} 
& $x_{1}$ & $x_{2}$ & $x_{3}$ & $x_{4}$ & $x_{5}$ & $x_{6}$ & $x_{7}$ \\
\hline$z_{1}$ & 0 & 0 & 0 & 1 & 1 & 1 & 1 \\
\hline$z_{2}$ & 0 & 1 & 1 & 0 & 0 & 1 & 1 \\
\hline$z_{3}$ & 1 & 0 & 1 & 0 & 1 & 0 & 1 \\
\hline
\end{tabular}

Then we obtain :

$$
\begin{aligned}
& z_{1}=x_{4}+x_{5}+x_{6}+x_{7} \\
& z_{2}=x_{2}+x_{3}+x_{6}+x_{7} \\
& z_{3}=x_{1}+x_{3}+x_{5}+x_{7}
\end{aligned}
$$

Therefore, to isolate the faulty sensor we apply the KLD test on the components $z_{1}, z_{2}, z_{3}$. For example if $x_{1}$ is faulty, we will detect the fault only on $z_{3}$ and not on $z_{2}$ and $z_{1}$ so the fault signature is '001' that corresponds as seen in Table 1 to the signal $x_{1}$. If $x_{7}$ is faulty, the three components $z_{1}, z_{2}$, $z_{3}$ are affected by the fault and can be detected by the KLD test, so the fault signature will be '111' that corresponds to the signal $x_{7}$.

Then using this method we can isolate the faulty sensor whatever the fault amplitude without any ambiguity. The main steps of the fault isolation procedure are shown in the flow-chart depicted in Fig.3.

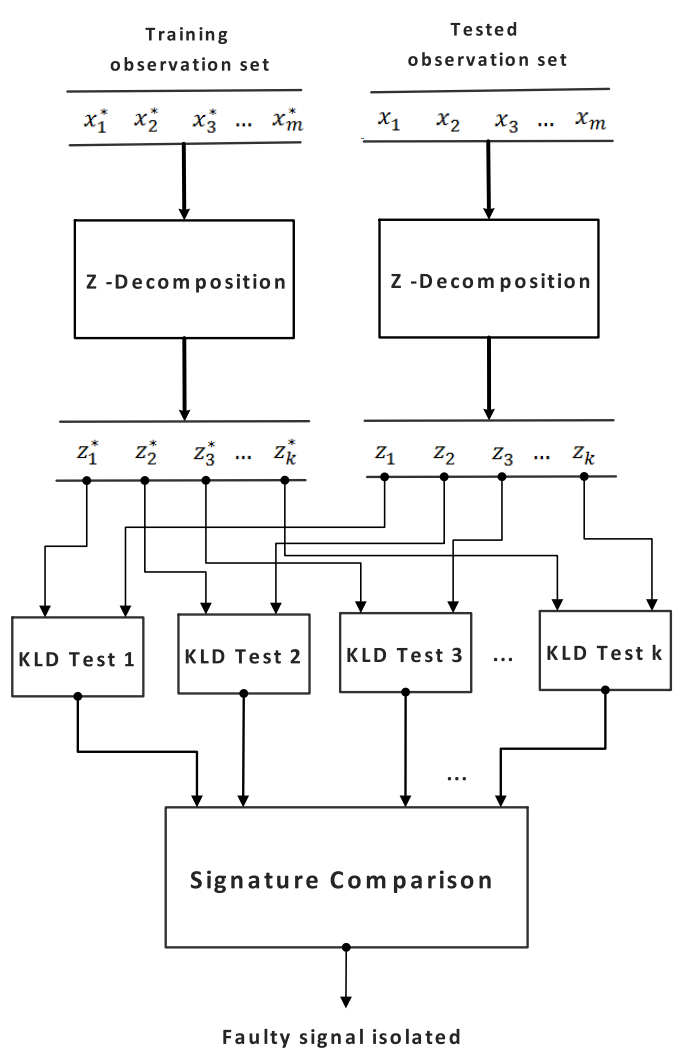

Fig. 3. Fault Isolation Procedure Flowchart

\section{RESULTS AND MODEL VALIDATION}

\subsection{Application to a numerical example}

We consider a system of $m=7$ variables defined as follows:

$$
\begin{array}{cc}
x_{1}(i) \sim \Gamma(1,2), & x_{2}(i) \sim \Gamma(4,5), \\
x_{3}(i) \sim \Gamma(1.5,3), & x_{4}(i)=2 * x_{1}(i), \\
x_{5}(i) \sim \Gamma(3,2), & x_{6}(i)=3 * x_{2}(i), \\
x_{7}(i) \sim \Gamma(7,5)
\end{array}
$$


This example is used for satisfying the theoretical assumption of the multivariate Gamma distribution of the data. We form a matrix $X$ of $N=10^{6}$ rows/samples, $X=\left(x_{1}, x_{2}, \ldots, x_{7}\right)$. Obviously, diagonalizing the covariance matrix of $X$ leads to 7 eigenvalues:

$\lambda=\left[\begin{array}{lllllll}998 & 150 & 20 & 13 & 10 & 0 & 0\end{array}\right]$.

For our study, we consider the first component $t_{1}^{*}$ where the corresponding eigenvalue is the biggest one. Then, we obtain $t_{1}^{*}=X p_{1}^{*}$, which summarizes the information contained into $X$. Then, the probability density of $t_{1}^{*}$ is estimated as the reference distribution and the probability density of the faulty $t_{1}$ is estimated as the test distribution. The fault affects only the variable $x_{6}$.

Firstly, to validate the fault detection procedure, a gain fault is added on $x_{6}$ with an amplitude 0.05 and $S N R=40 d B$. Fig.4 displays the KLD computed on $t_{1}$ for 50 realisations: first 25 without fault, and the last 25 with fault $(a=0.05)$. As seen in this figure the proposed method can detect easily the fault occurrence without any ambiguity.

Now, to locate the faulty signal, our proposed procedure is applied. In this case $K_{z}=3$ and the KLD is computed on the three components $z_{1} z_{2} z_{3}$. As seen in Fig.5 the fault is detected only on $z_{1}$ and $z_{2}$ and not on $z_{3}$. Then the fault signature obtained is 110 which corresponds to a fault on $x_{6}$.

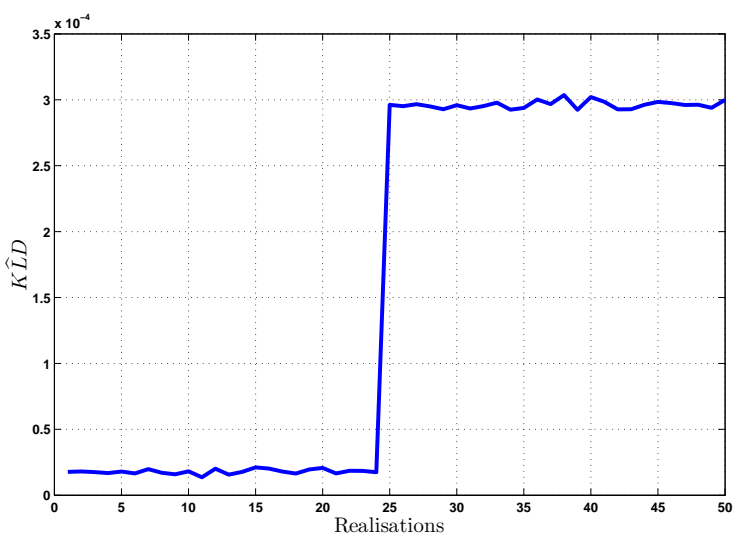

Fig. 4. Fault detection results for simulated data

\subsection{Application to accelerometers measurements}

In this section, the proposed procedure is evaluated with experimental vibration data from the Case Western Reserve University (CWRU, Bearing Data Center) [15]. The goal is to detect, isolate and estimate a vibration sensor fault.

According to the description given by the provider of the test data, the test bed consists of a 3 hp motor, a torque transducer/encoder, and a dynamometer. The eight vibration
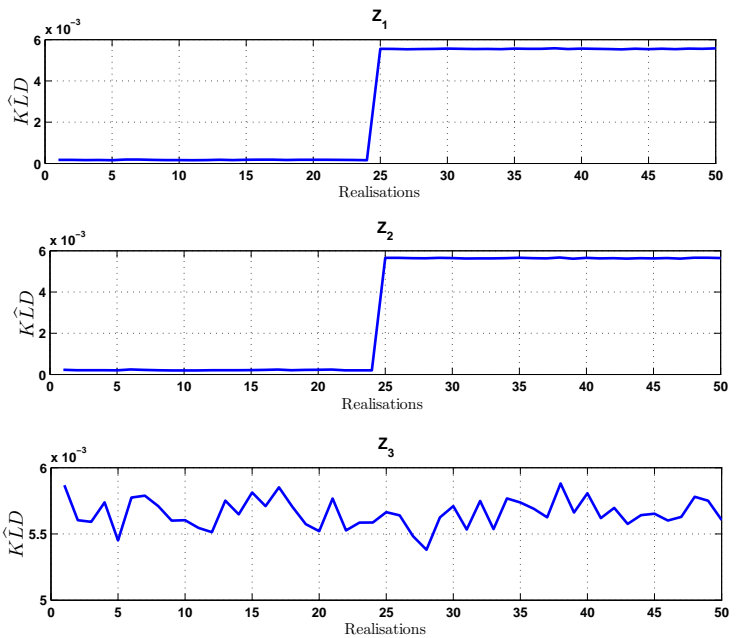

Fig. 5. Fault isolation results

signals (4 at drive end and 4 at fan end) are acquired, at a sampling frequency of $12 \mathrm{KHz}$ during 10 seconds. They are measured for 4 different speeds $(1797,1772,1750$ and $1730 \mathrm{rpm})$. The data matrix $X$ built with these 8 signals is processed through PCA. For the fault localisation, as we have 8 signals, the number of components obtained from the Z-decomposition is $K_{z}=4$. In Table 2, we show the Z-decomposition and the fault signatures.

Table 2. Experimental Fault signature results

\begin{tabular}{ccccccccc} 
& $x_{1}$ & $x_{2}$ & $x_{3}$ & $x_{4}$ & $x_{5}$ & $x_{6}$ & $x_{7}$ & $x_{8}$ \\
\hline$z_{1}$ & 0 & 0 & 0 & 0 & 0 & 0 & 0 & 1 \\
\hline$z_{2}$ & 0 & 0 & 0 & 1 & 1 & 1 & 1 & 0 \\
\hline$z_{3}$ & 0 & 1 & 1 & 0 & 0 & 1 & 1 & 0 \\
\hline$z_{4}$ & 1 & 0 & 1 & 0 & 1 & 0 & 1 & 0 \\
\hline
\end{tabular}

Then we obtain :

$$
\begin{gathered}
z_{1}=x_{8} \\
z_{2}=x_{4}+x_{5}+x_{6}+x_{7} \\
z_{3}=x_{2}+x_{3}+x_{6}+x_{7} \\
z_{4}=x_{1}+x_{3}+x_{5}+x_{7}
\end{gathered}
$$

To evaluate the fault detection and isolation procedure, we add a gain fault to the signal $x_{6}$ with an amplitude $a=0.01$. On the las 25 realisations, applying the KLD test on the first principal component, the fault occurrence is detected as displayed in Fig.6 .

Now to isolate the faulty signal we apply our procedure. In Fig.7, the result of the KLD-test on the 4 components $z_{1} z_{2} z_{3}$ $z_{4}$ is shown. As it can be seen in this figure, only the KLD on $z_{2}$ and $z_{3}$ have a significant variation at the $25^{\text {th }}$ realisation. 
Therefore the fault signature is "0110" that corresponds to a fault on $x_{6}$ as confirmed in Table 2 .

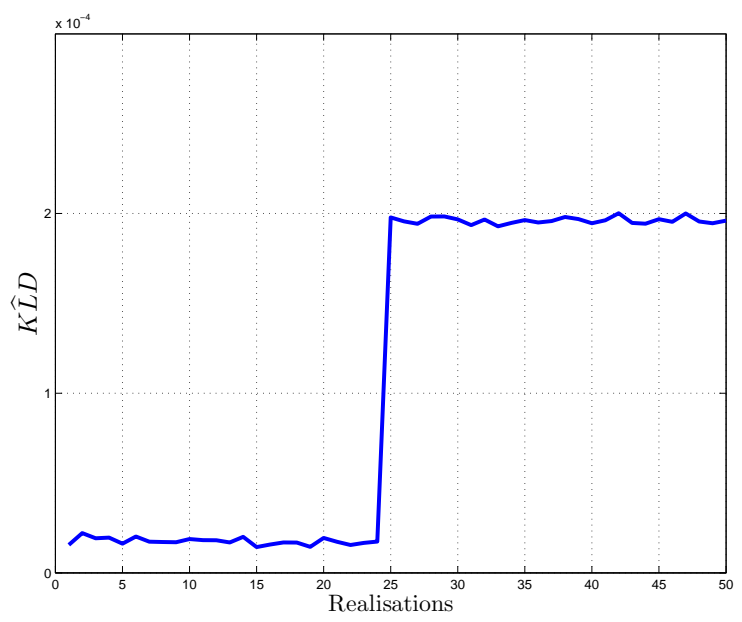

Fig. 6. Fault Detection results for experimental data
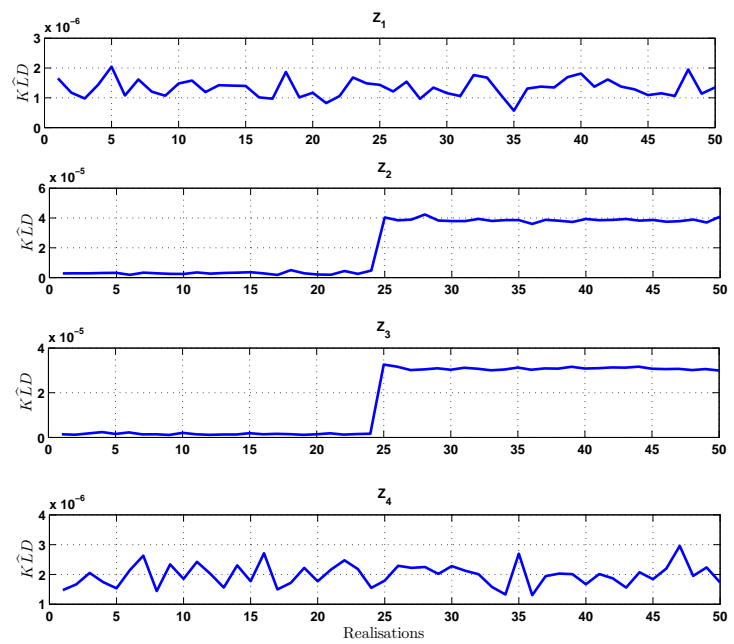

Fig. 7. Accelerometers fault isolation results

\section{CONCLUSION}

In this paper we have developed a multisensor fault detection and isolation method using the Kullback-Leibler Divergence, the PCA-based data representation and the Z-decomposition. The fault detection and estimation procedure has been successfully validated with experimental vibration signals collected on accelerometers used for health monitoring of bearings in electrical machines. The results show a high efficiency of the proposed method.

\section{REFERENCES}

[1] J.C. Trigeassou, Electrical Machines Diagnosis, Wiley, ISTE, 2011.

[2] C.K. Mechefske and J. Mathew, "Fault detection and diagnosis in low speed rolling element bearings part I: The use of parametric spectra," Mechanical Systems and Signal Processing, vol. 6, no. 4, pp. 297 - 307, 1992.

[3] R. Isermann, Fault-Diagnosis Systems: An Introduction from Fault Detection to Fault Tolerance, Berlin Heidelberg: Springer, 2006.

[4] S. Alag, A. M. Agogino, and M. Morjaria, "A methodology for intelligent sensor measurement, validation, fusion, and fault detection for equipment monitoring and diagnostics," Artificial Intelligence for Engineering Design Analysis and Manufacturing, vol. 15, no. 4, pp. 307-320, September 2001.

[5] P.H. Ibarguengoytia, L.E. Sucar, and S. Vadera, "Real time intelligent sensor validation," IEEE Transactions on Power Systems, vol. 16, no. 4, pp. 770-775, 2001.

[6] J. Harmouche, C. Delpha, D. Diallo, and Y. Le-Bihan, "Statistical approach for non-destructive incipient damage detection and characterisation using Kullback-Leibler divergence," IEEE Transaction on Reliability, vol. 65, no. 3, pp. 1360-1368, 2016.

[7] Q. Wang, Y. Zhang, L. Cai, and Y. Zhu, "Fault diagnosis for diesel valve trains based on non-negative matrix factorization and neural network ensemble," Mechanical Systems and Signal Processing, vol. 23, no. 5, pp. 1683 - 1695, 2009.

[8] S. Yin, X. Ding, X. Xie, and H. Luo, "A review on basic data-driven approach for industrial process monitoring," IEEE Transactions on Industrial Electronics, vol. 61, no. 11, pp. 6418-6428, 2014.

[9] I.T. Jollife, Principle Component Analysis, Second Edition, Aberdeen U.K, 2002.

[10] F. Harrou, M. N. Nounou, H. N. Nounou, and M. Madakyaru, "Statistical fault detection using PCA-based GLR hypothesis testing," Journal of loss prevention in the process industries, vol. 26, pp. 129-139, 2013.

[11] S. Kullback and R.A. Leibler, "On information and sufficiency," The Annals of Mathematical Statistics, vol. 22, no. 1, pp. 79-86, 1951.

[12] J. Harmouche, C. Delpha, and D. Diallo, "Incipient fault detection and diagnosis based on Kullback-Leibler divergence using principal component analysis: Part I," Elsevier Journal on Signal Processing, vol. 94, pp. 278-287, January 2014.

[13] L. T. B. Nguyen, M. Djeziri, B. Ananou, M. Ouladsine, and J. Pinaton, "Fault prognosis for batch production based on percentile measure and gamma process: Application to semiconductor manufacturing," Journal of Process Control, vol. 48, pp. $72-80,2016$.

[14] A. Youssef, C. Delpha, and D. Diallo, "Performances theoretical model-based optimization for incipient fault detection with KL divergence," in European Signal Processing Conference (EUSIPCO 2014), Lisbon, Portugal, September 1-5 2014, IEEE, pp. 466-470.

[15] Case Western Reserve University, "Bearing data centre," http://csegroups.case.edu/bearingdatacenter/ pages/downloaddata-file/, 2016, [Online; accessed May-2016]. 